\title{
Beyond the "Dynamic range" approach In noise evaluation for Terahertz Time domain spectrometers
}

\author{
Clémence Muller ${ }^{1}$, Mélanie Lavancier ${ }^{1}$, Jeyan Bichon ${ }^{1}$, Théo Hannotte, \\ Jean-François. Lampin ${ }^{1}$, Sophie Eliet ${ }^{1}$ and Romain Peretti ${ }^{1}$ \\ ${ }^{1}$ Univ. Lille, CNRS, Centrale Lille, Univ. Polytechnique Hauts-de-France, \\ UMR 8520 IEMN - Institut d'Electronique de Microélectronique et de Nanotechnologie, F-59000 Lille, France
}

\begin{abstract}
THz - TDS is used for a myriad of purposes. Still today, the usual way to compare different system in terms of signal to noise ratio is to look at the dynamic range. We propose here a more reliable method based on statistics and will show results on two systems (Toptica and Menlo).
\end{abstract}

\section{INTRODUCTION}

$\mathrm{T}$ HE goal of every spectroscopy is to extract information from the recorded data. This information lies in models fitting a transfer function from a measured reference to a measured sample $[1,2,3]$. This comparison is straightforward in frequency-domain spectroscopy since the transfer function is used in a multiplication. In addition, the noise is often an uncorrelated white noise of which envelop serves as weighing function for the fit.

However, as well explained in [4, 5], the specificity of THz-TDS, the data are in the time domain and the models are still the same: in the frequency domain. It follows that the multiplications by the transfer function in the frequency domain become convolutions by a transfer function in the time domain. Therefore, the non-correlated hypothesis for noise is never true. E.g. for a slab sample, the model transforms the reference pulse into several Fabry-Perrot echoes, each of them getting the same, consequently correlated, noise. Thus, the weighting function does not apply any more, but one needs to use the noise correlation matrix as weighting in the fit. It means that the knowledge of the noise both correlated and uncorrelated is extremely important.

Impact of the noise on THz-TDS measuremnts is a long haul effort [6] and we propose here to use a very common but still not standardized methods based on statistics and repetition of the measurements.

\section{RESULTS}

The datasheet of the THz-TDS systems includes dynamic range data that compare the signal at the peak frequency to the noise at frequency where the system does not operate. This clearly help making the difference from a first generation system with the new ones. However, it is not a clear figure on how reliable will be your measurements. To go further towards this direction, we propose a statistical approach in measuring several time traces and compare their mean value (signal) and standard deviation (noise) in the time (fig. 1 ) and frequency domain (fig. 2).

As seen on fig. 2, the dynamic range vision tremendously underestimate the noise in the range of high signal to noise ratio. Here we plotted the raw and corrected data from measurements of five 100 ps time long traces (5000 average). One of the reason is that noise does not only come from the variation of current from the detector but as well from imprecision on the delay line [7], parasitic low frequency emission and variation of the power of the excitation laser. To take it into account, we corrected the time traces from a fixed delay (mean square optimization) after a low frequency filtering ( $1^{\text {st }}$ correction), and further away using a correction term proportional to the signal and to its second derivative to take into account the variation of the laser $\left(2^{\text {nd }}\right.$ correction).

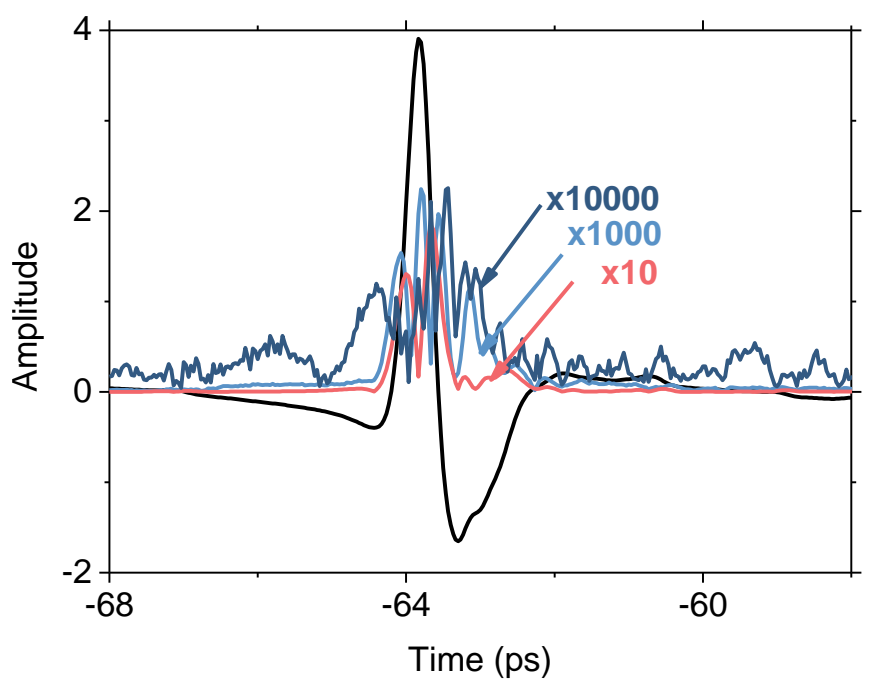

Fig. 1. Time domain signal (mean value) and noise (standard deviation) of TDS measurements without correction (no correction), with a delay shift correction after a high pass filter $\left(\mathbf{1}^{\text {st }}\right.$ correction), with in addition a correction term proportional to the signal and the second derivative of the signal $\left(2^{\text {nd }}\right.$ correction $)$.

This shows that first the noise cannot simply be considered as a dark noise uncorrelated one. In addition, it shows that after few corrections it is possible to decrease the noise tremendously even bellow the usual dynamic range vision. 


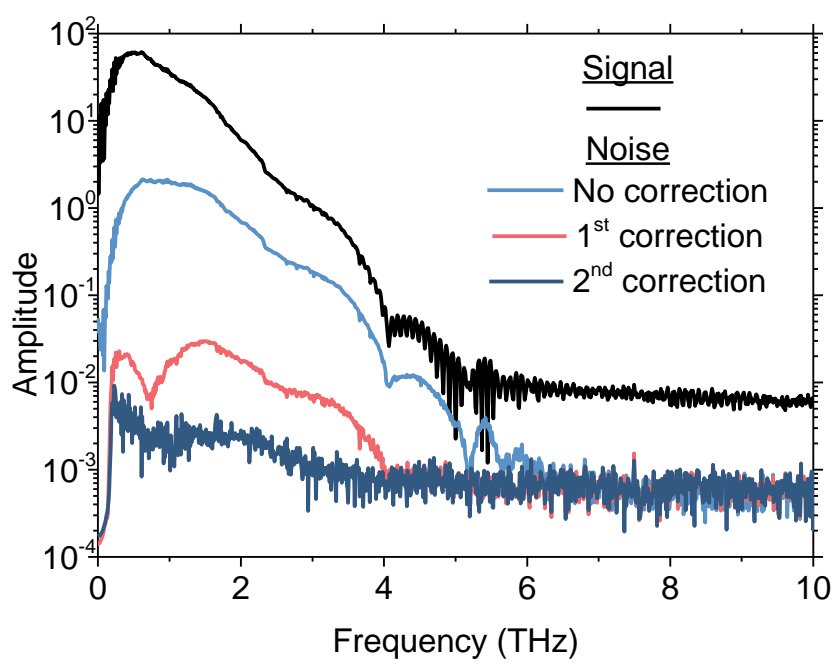

Fig. 1. Time domain signal (mean value) and noise (standard deviation) of TDS measurements without correction (no correction), with a delay shift correction after a high pass filter ( $1^{\text {st }}$ correction), with in addition a correction term proportional to the signal and the second derivative of the signal $\left(2^{\text {nd }}\right.$ correction).

\section{SUMMARY}

THz-TDS is one of the major assets of the $\mathrm{THz}$ range thanks to the compactness, robustness, broadband and high signal to noise ratio of this system. Today the use of the dynamic range as standard for signal to noise ratio does not meet the requirement for a qualitative information extraction from the recorded data. We proposed here a classic statistical method to compare two systems and show that additional corrections can improve the signal to noise ratio by more than $50 \mathrm{~dB}$.

\section{ACKNOWLEDGMENTS}

The authors want to thanks the "Région Hauts-de-France" for the International Chair of Excellence "ThOTroV, the "TeraStoVe" pluridisciplinary project from I-site ULNE, the welcome talent grant "NeFiStoV" from the European Metropole of Lille (MEL); and the "Agence Nationale de la Recherche" for the STEPSON ANR-20-CE42-0002 grant?

\section{REFERENCES}

[1]Romain Peretti, Martin Micica, Sergey Mitryukovskiy, Kevin Froberger, Sophie Eliet, Mathias Vanwolleghem, and Jean-François Lampin. Modeling and parameter retrieving in time domain spectroscopy of material and metamaterial. In Nonlinear Optics and its Applications 2018, volume 10684, page 106840P. International Society for Optics and Photonics, 2018

[2]R. Peretti, S. Mitryukovskiy, F. Kevin, and J. Lampin. Retrieving material and metamaterial parameters directly from time-domain spectroscopy time trace. In 2018 43rd International Conference on Infrared, Millimeter, and Terahertz Waves (IRMMW-THz), pages 1-2, Sept 2018

[3]Romain Peretti, Sergey Mitryukovskiy, Kevin Froberger, Anis Mebarki, Sophie Eliet, Mathias Vanwolleghem, and Jean-François Lampin. Thz-tds time-trace analysis for the extraction of material and metamaterial parameters IEEE Transactions on Terahertz Science and Technology, 9(2):136-149, March 2019.

[4]Laleh Mohtashemi, Paul Westlund, Derek G. Sahota, Graham B. Lea, Ian Bushfield, Payam Mousavi, and J. Steven Dodge. Maximum-likelihood parameter estimation in terahertz time-domain spectroscopy. Opt. Express, 29(4):4912-4926, Feb 2021.

[5]J Steven Dodge, Laleh Mohtashemi, Paul Westlund, Payam Mousavi, and Derek G Sahota. A maximum-likelihood analysis framework for terahertz time-domain spectroscopy. In 2020 45th International Conference on Infrared, Millimeter, and Terahertz Waves (IRMMW-THz), pages 1-1. IEEE, 2020.

[6]Lionel Duvillaret, Frédéric Garet, and Jean-Louis Coutaz. Influence of noise on the characterization of materials by terahertz time-domain spectroscopy. JOSA B, 17(3):452-461, 2000 .

[7] Arno Rehn, David Jahn, Jan. C. Balzer, and Martin Koch. Periodic sampling errors in terahertz time-domain measurements. Opt. Express, 25(6):6712-6724, Mar 2017. 